\title{
Sources and characteristics of block falls at the Martian north polar scarps
}

\author{
Shu Su ${ }^{1}$, Lida Fanara ${ }^{1,2}$, Xin Zhang ${ }^{3}$, Klaus Gwinner ${ }^{2}$, Ernst Hauber ${ }^{2}$, and Jürgen Oberst ${ }^{1,2}$ \\ ${ }^{1}$ Institute of Geodesy and Geoinformation Science, Technical University of Berlin, Berlin, Germany (shu.su@campus.tu-berlin.de) \\ ${ }^{2}$ Institute of Planetary Research, DLR, Berlin, Germany \\ ${ }^{3}$ College of Surveying and Geoinformatics, Tongji University, Shanghai, China
}

\begin{abstract}
We are exploring mass wasting at the scarps of the Martian North Polar Layered Deposits (NPLD) by probing into collapsed ice-fragments using multi-temporal HiRISE images. We apply change detection techniques to the images in areas of steep scarps and then determine sizes and volumes of the ice-fragments using boundary mapping in image pyramids. Our aim is to map the sources of block fall events through time, estimate volumes of displaced material and investigate their seasonality in order to better understand the behavior of the ice scarps and gain insights into their evolution.
\end{abstract}

\section{Introduction}

The steep scarps at the boundary of the NPLD have shown evidence of mass wasting in recent decades [1]. In particular, dust avalanches and ice-block falls, are probably related to thermoelastic stresses [2]. Ice-block falls are playing a significant role of active erosion at the north polar scarps of Mars [3,4,5]. Data from the High Resolution Imaging Science Experiment (HiRISE), including multi-temporal images with scales of up to $\sim 0.25 \mathrm{~m} /$ pixel, make it possible to identify small-scale changes [6]. Fanara et al. (2019) estimated the erosion rate of a scarp by detecting block falls and have found seven areas with similarly fractured scarps [5], from which ice-fragments detach and finally fall as ice-blocks. Figure 1 shows three sets of examples from different scarps, where icefragments have been detected in Mars Year 31 and have disappeared in Mars Year 32 (red arrows). The red circle in Figure $1 a$ indicates the ice-block falls from the collapsed ice-fragment. Considering the active erosion during the past decades, we want to investigate the sources of the block falls and map them through time. 

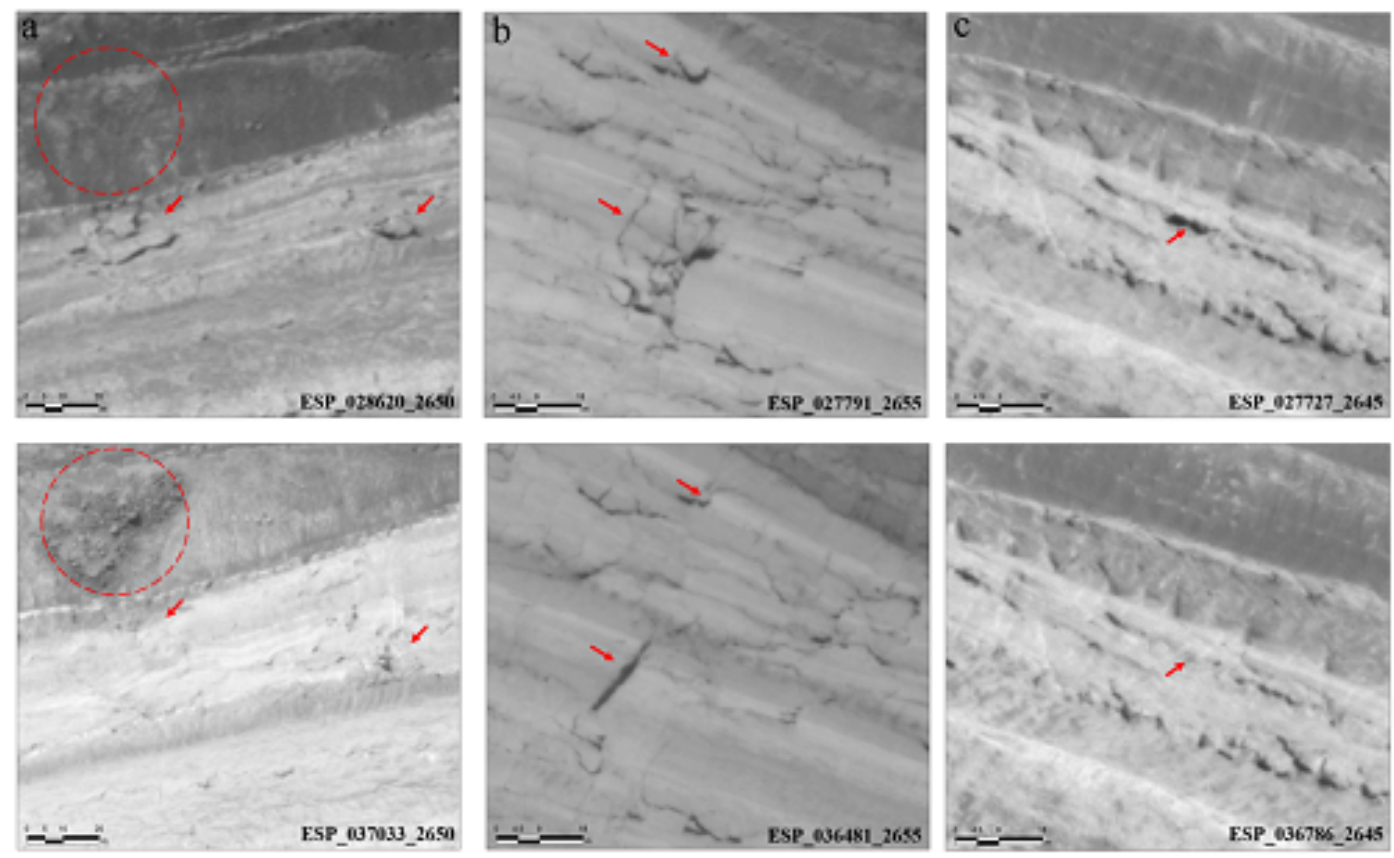

Figure 1. Three sets of images from different scarps between Mars Year 31 (first row) and Mars Year 32 (second row) show collapsed ice-fragments (red arrows). The red circle shows a resulting block fall event.

\section{Method}

Based on two ortho-rectified HiRISE images processed by Ames Stereo Pipeline (ASP) at different times, we detect the collapsed ice-fragments' areas. We focus on images taken during the summer of Mars because the covering $\mathrm{CO}_{2}$ ice sublimates in spring and then the scarps will show distinct textures in summer. Before the ice-fragments collapse, they have shadows due to the solar incidence. While after the ice-fragments fall down, the original shadows disappear, allowing us to detect the active erosion.

We often find that for ortho-rectified images, co-registration must be refined. For this, we use image matching techniques. First, we use the normalized cross-correlation to find the approximate corresponding position of two images. Then, we divide both images into multiple tiles to avoid geometric deviations in different areas over the entire image. Regarding regional changes due to collapsed ice-fragments, intensity-based matching methods are sensitive, while optimized feature matching can work well. So, we use feature-based matching method combined with affine transform on all tiles to align them. In order to reduce radiometric differences caused by illumination, atmosphere and other conditions, we apply Wallis filter for radiometric normalization. Lastly, we obtain the difference image by subtraction and set a threshold to get the binary difference image, which shows the changed area.

On the difference image, the obvious discrepancy between the two images are the shadow areas. However, it is not always easy to distinguish the complete boundaries of the ice-fragments. So we intend to detect fuzzy edges via image pyramids and contrast enhancement in order to calculate the fragments' areas.

The height of the ice-fragments are also critical for calculating the volume of the material loss. The vertical precision of HiRISE DEM is about tens of centimeters [6]. However, most of the fragments' heights are $\sim 1 \mathrm{~m}$, thus it is hard to determine elevation differences through DEMs. Therefore, we will combine the solar incidence angle with the shadow length of the ice-fragments to calculate their 
height.

\section{Discussion}

Our research focuses on the investigation of the source areas of block falls at the NPLD. The change detection method results in maps of the areas of active erosion through time. This way we derive important temporal and spatial constraints as well as volume estimations for the ongoing block fall activity. Ultimately we want to analyze the scarp activity based on the correlation of our results with seasonal and morphological parameters to help understand the ice behavior and the evolution of the NPLD scarps.

\section{Acknowledgements}

The first author thanks China Scholarship Council (CSC) for the financial support to study in Germany.

\section{References}

[1] Russell, P. S., et al. 5th ICMPSE. Vol. 1623. 2011.

[2] Byrne, S., et al. EPSC. Vol. 11. 2017.

[3] Herkenhoff, K. E., et al. Science. 317.5845 (2007): 1711-1715

[4] Russell, P. S., et al. 8th Intern. Conf. on Mars. Vol. 1791. 2014.

[5] Fanara, L., et al. 113434. 2019.

[6] McEwen, Alfred S., et al. JGR. 112. E5. 2007. 